\title{
Aleitamento materno em prematuros: atuação fonoaudiológica baseada nos pressupostos da educação para promoção da saúde
}

\author{
Breastfeeding in premature babies: speech-language and audiology \\ performance based on education for health promotion concepts
}

\author{
M aria da Conceição Carneiro Pessoa de Santana ${ }^{1}$ \\ Bárbara N iegia Garcia de Goulart² \\ Brasília M aria Chiari ${ }^{3}$ \\ Adriana de $\mathrm{M}$ edeiros $\mathrm{M} \mathrm{elo}^{4}$ \\ Érika Henriques de Araújo Alves da Silva ${ }^{4}$
}

\footnotetext{
${ }^{1}$ Serviço deFonoaudiologia, M aternidadeEscolaSanta M ônica, Universidade Estadual deCiências da Saúdedo Estado deAlagoas. Av. Comendador Leão, $s / n$, Poço. 57025-200 Maceió AL. cpessoafono@yahoo.com.br ${ }^{2}$ UniversidadeFederal do Rio Grande do Sul.

${ }^{3}$ UniversidadeFederal de São Paulo.

${ }^{4}$ Universidade Estadual de Ciências da Saúde do Estado deAlagoas.
}

Abstract This paper reports an experience of a speech-languageand audiology (SLP) team based on health promotion and education to contribute for the maintainance of exclusive breastfeeding in premature newborns in a high-risk maternity. In the process, a multiprofessional group and individual strategies were combined, producing dialogic settings along with puerperals and their families. Exclusive breastfeeding increased comparing with the last three years before these approach and greater participation of the team and relatives involved in these activities since the pregnant admission until discharge. The analisys of thestrategies used allowed the team to identify the most effective actions to improve longer lasting exclusive breastfeeding and those that ought to be reformulated. From a theoretical and methodological point of view, this experience also allowed to observe the limits and possibilities of the actions that are related to closer areas of scientific knowledgeand theeffective promotion of exclusive breastfeeding in transdisciplinary activities. Considering health promotion and education as a discipline, it means that the efforts are highly directed to act on thepeopleknowledgeand self-care, mainly to contribute to the development of critical judgment and ability to decide what would be better and possible in health care and in managing of their own lives.

Key words Breastfeeding, Premature, H ealth education, Health promotion, Disease prevention
Resumo 0 artigo relata experiência de atuação fonoaudiológica baseada nos pressupostos da educação em saúde direcionada à promoção do aleitamento exclusivo em prematurosinternados em uma maternidade de referência em alto risco. No trabaIho desenvolvido, estratégias multiprofissionais realizadas com grupos e de forma individualizada foram combinadas, criando espaços dialógicos com as puérperas e seus familiares e a prevalência de aleitamento materno exclusivo aumentou nos últimos três anos na enfermaria. H ouve grande participação dos envolvidos nas atividades propostas, desde a admissão da puérpera na enfermaria ao processo da alta hospitalar. A estratégia adotada permitiu detectar as intervenções mais efetivas eas que deverão ser reformuladas com toda equipe. Do ponto de vista teórico e metodológico, a experiência também permitiu observar os limites epotencialidades de ações que aproximam a área do saber científico e a promoção do aleitamento materno efetiva em atividades transdisciplinares. Conside rar a educação em saúde como disciplina de ação significa dizer que o trabalho é dirigido para atuar sobre o conhecimento das pessoas, para que elas desenvolvam juízo crítico e capacidade de intervenção sobre suas próprias vidas e sobre o ambiente com o qual interagem e, assim, criarem condições para se apropriarem de sua própria existência. Palavras-chave Aleitamento materno, Prematuro, Educação em saúde, Promoção da saúde, Pre venção de doenças 
Introdução

Entre os anos setenta e oitenta, a fonoaudiologia foi inserida no sistema público por meio das Secretarias de Educação e Saúde. O s serviços geralmente eram estruturados como consultórios, marcados por práticas reabilitadoras ${ }^{1}$. Inicialmente, o fonoaudiólogo tentou adotar a estrutura de atendimento clínico-privado no sistema público, com resultados pouco satisfatórios. A partir disso, novos caminhos foram buscados, a fim de que um melhor desempenho fosse obtido nos vários níveis de atenção à saúde. $A$ atuação fonoaudiológica no contexto da saúde pública implica uma mudança marcada por práticas assistencialistas e reabilitadoras para a capacidade de elaborar e efetivar ações que visem sua solução, bem como adotar medidas preventivas cabíveis, prestando um atendimento de qualidade à populaçãa ${ }^{2}$.

Ao longo dos anos, pesquisas no campo da fonoaudiologia comunitária tornaram-se relevantes, com a finalidade de oferecer contribuição para definir melhor o papel elugar do fonoaudiólogo junto à promoção da saúde da população de maneira reflexiva, consciente, responsável e atuante ${ }^{3}$.

O profissional de saúde pública deve ter por objetivo a promoção, prevenção e recuperação da saúde fonoaudiológica da população, tendo interesse nos distúrbios que afetam um grupo específico, mas aplicando medi das de caráter comunitário ${ }^{46}$. Paraisso, épreciso conhecer as condições de saúde deste grupo, por meio da caracterização da população usuária dos serviços coletivos, mediante estudos epidemiológicos, a fim de elaborar políticas adequadas à populaçãa, 5,6. A ação do fonoaudiólogo no serviço público requer capacidade de percepção das alterações fonoaudiológicas que acometem a população em geral, como também das peculiaridades e dinamicidade da demanda do local onde o profissional está lotado ${ }^{4}$. Os agravos à comunicação humanajáforam largamentedescritos. Há queevoluir no conhecimento mais detalhado de questões ligadas aos fatores de risco para tais alterações, além dos efeitos destes para o sujeito ${ }^{5,6}$.

Esteartigo se propõe a contribuir com subsídios para a atuação fonoaudiológica diante do aleitamento materno em prematuros, sob a ótica da educação em saúde, por meio de relato de experiência erevisão da literatura indexada epublicada nas bases de dados da biblioteca virtual em saúde (BVS) e SciELO.

\section{Educação em saúde: emergência deum novo} discurso

O conceito dehumanização das práticas e da atenção à saúde está na pauta de discussões mundo afora há várias décadas e nos últimos anos vem ganhan do destaque na literatura científica nacional, principalmentenas publicações ligadas à saúdecoletiva ${ }^{6}$.

Dentre os diversos espaços dos serviços de saúde, destacam-se os de atenção básica como um contexto privilegiado para desenvolvimento de práticas educativas em saúde devido à particularidade destes serviços, caracterizados pela maior proximidade com a população e a ênfase nas ações preventivas e promocionais ${ }^{7}$. Além disso, os serviços de atenção básica precisam apropriar-se de uma tecnologia de alta complexidade que envolve conhecimentos, habilidades etécnicas, dentre as quais é possível reconhecer a educação em saúde. Relacionando as funções de um médico de atenção básica, 0 autor destaca prestar atenção preventiva, curativa e reabilitadora, ser comunicador e educador em saúde ${ }^{8}$.

Além das melhorias das condições gerais de vida, a implantação de postos de saúde e de puericultura foi decisiva na melhoria dos indicadores de aleitamento materno (AM), pois nesses locais é possível estabelecer ações diretamente desenvolvidas pel o pediatra esua equipejunto às mães e futuras mães, caracterizando o AM como uma das maiores e mais importantes iniciativas de promoção à saúde?.

A educação em saúde constitui um conjunto de saberes e práticas orientadas para a prevenção de doenças e promoção da saúde. Trata-se deum recurso por meio do qual o conhecimento cientificamente produzido no campo da saúde, intermediado pelos profissionais de saúde, atinge a vida cotidiana das pessoas, uma vez que a compreensão dos condicionantes do processo saúde-doença oferece subsídios para a adoção de novos hábitos e condutas de saúde ${ }^{10}$.

A iniciativa dos profissionais em inserir-seem serviços de saúde que prestavam assistência às classes populares se deu integrada a projetos mais amplos, dentre os quais predominava a metodologia da educação popular. Assim sendo, esta metodologia foi assimilada pelo movimento dos profissionais, constituindo seu elemento estruturante fundamental, e tem priorizado a relação educativa com a população, rompendo com a verticalidade da relação profissional-usuário. Valorizam-se as trocas interpessoais, as iniciativas da população e usuários e, pelo diálogo, bus- 
cam-se a explicitação e compreensão do saber popular. Esta metodologia contrapõe-se à passividade usual das práticas educativas tradicionais. O usuário é reconhecido como sujeito portador de um saber sobre o processo saúde-doença-cuidado, capaz de estabelecer uma interlocução dialógica com o serviço de saúde e de desenvolver uma análise crítica sobre a realidade e o aperfeiçoamento das estratégias de luta e enfrentamento. Pela potencialidade desta metodologia, vislumbra as experiências de educação popular como forma de superação do fosso cultural entre os serviços de saúde e a população assistida ${ }^{7}$.

A despeito da emergência de um novo discurso no campo da educação em saúde, prevalecem as práticas educativas hegemônicas. Da convivência entre as práticas emergentes e hegemônicas, é possível delinear dois modelos de práticas de educação em saúde, que podem ser referidos como modelo tradicional e modelo dialógico. Estes se encontram em pólos extremos, sendo possível reconhecer modelos intermediários ${ }^{11}$.

0 modelo tradicional, historicamente hegemônico, focaliza a doença ea intervenção curativa e é fundamentado no referencial biologicista do processo saúde-doença, preconizando que a prevenção das doenças prima pela mudança de atitudes e comportamentos individuais. As estratégi as desta prática educativa em saúde incluem informações verticalizadas que ditam comportamentos a serem adotados para a manutenção da saúde ${ }^{5,6,12}$. Os usuários são tomados como indivíduos carentes deinformação em saú$\mathrm{de}^{11}$. Desta maneira, a relação estabelecida entre profissionais eusuários éessencialmenteassimé trica, uma vez que um detém um saber técnicocientífico, com status de verdade, enquanto o outro precisa ser devidamente informado. A comunicação profissional-usuário caracteriza-se pelo caráter informativo, na qual o primeiro, assumindo uma atitu de paternalista, explicita ao segundo hábitos e comportamentos saudáveis, o que fazer e como fazer para a manutenção da saúde. Pressupõe-se, ainda, que a partir da informação recebida, os usuários serão capazes de tomar decisões para a prevenção de doenças e agravos, bem como poderão assumir novos hábitos e condutas ${ }^{12}$.

É importantedestacar quea principal crítica a este modelo de educação tem sido a desconsideração dos determinantes psicossociais e culturais dos comportamentos no processo de saúde e doença ${ }^{5,6}$. Ao tomar os usuários como objeto das práticas educativas e carentes de um saber sobrea saúde, perde-se de vista que os comportamentos são orientados por crenças, valores, representações sobre o processo saúde-doença, todos estes representantes de formas outras de saber ${ }^{13}$.

A proposição depráticas educativas sensíveis às necessidades dos usuários insere-se no discurso emergentedeeducação em saúde: o modelo dialógico. Em oposição ao modelo tradicional, trabalha-se com a perspectiva de sujeitos das práticas de saúde ${ }^{14}$. Neste sentido, apresentamsedois princípios básicos na orientação das ações de saúde. Primeiramente, é necessário conhecer os indivíduos para os quais se destinam as ações de saúde, incluindo suas crenças, hábitos, papéis e as condições objetivas em que vivem. $O$ segundo princípio parte da premissa de que é preciso envolver os indivíduos nas ações, o que se contrapõe a sua imposição. Vale ressaltar que apenas com a participação comunitária é possível assegurar sustentabilidade e efetividade das ações de saúde ${ }^{11}$.

O modelo emergente de educação em saúde pode ser referido como modelo dialógico por ser o diálogo seu instrumento essencial. 0 usuário dos serviços é reconhecido sujeito portador de um saber, que embora diverso do saber técnicocientífico não é deslegitimado pelos serviços.

O objetivo da educação dialógica não é o de informar para a promoção da saúde, mas transformar saberes existentes. A prática educativa, nesta perspectiva, visa ao desenvolvimento da autonomia e da responsabilidade dos indivíduos no cuidado com a saúde, porém não mais pela imposição de um saber técnico-científico detido pelo profissional de saúde, mas sim pelo desenvolvimento da compreensão da situação de saúde. O bjetiva-se, ainda, queessas práticas educativas sejam emancipatórias. A estratégia valorizada por este modelo éa comunicação dialógi$\mathrm{ca}$, que visa à construção de um saber sobre 0 processo saúde-doença-cuidado que capacite os indivíduos a decidirem quais as estratégias mais apropriadas para promover, manter e recuperar sua saúde $\mathrm{e}^{15}$.

O Scientific Group on Research in H ealth Education (Grupo Científico sobre Pesquisa em Educação em Saúde), da Organização Mundial da Saúde (OMS), expandiu essa declaração ao afirmar que "os objetivos da educação em saúde são desenvolver nas pessoas o senso de responsabilidade pela sua própria saúde e pela saúde da comunidade a qual pertençam e a capacidade de participar da vida comunitária de uma maneira construtiva"16.

A partir do diálogo e intercâmbio de saberes técnico-científicosepopulares, profissionaiseusu- 
ários podem construir de forma compartilhada um saber sobre o processo saúde-doença. Este compromisso e a vinculação com os usuários possibilitam o fortalecimento da confiança nos serviços. Por esta circunstância, o modelo dialógico tem sido associado a mudanças duradouras de hábitos e de comportamentos para a saúde, visto serem ocasionados não pela persuasão ou autoridade do profissional, mas pela construção de novos sentidos e significados individuais e coletivos sobre o processo saúde- doença-cuidado ${ }^{15}$.

\section{Relato de experiência}

Junto à equipe da enfermaria canguru, da M aternidade Escola Santa M ônica, referência em alto risco, em Maceió (AL), um processo de trabalho direcionado ao binômio mãe-bebê foi desenvolvido a partir do ano de 2003, baseado no modelo dialógico da educação em saúde. A genitora éencaminhada pela unidade de cuidados intermediários, unidade de terapia intensiva ou pelo alojamento conjunto. Ao ser admitida na enfermaria canguru, esta mãe tem a oportunidade de realizar um diálogo também com o serviço de fonoaudiologia. N esse momento, uma comunicação começa a ser estabel ecida edados são coletados pelo profissional, que considera todos os saberes, principal mente no que se refere aos mitos, às crenças e experiências relativas ao aleitamento materno. Por meio dessa comunicação dialógica, um diagnóstico da situação começa a ser realizado. São detectadas as possíveis mudanças necessárias e estas construídas por meio do diálogo e intercâmbio de saberes técnico-científicos e populares. Essa estratégia visa à construção de novos sentidos e significados (individuais e coletivos) sobre o estabelecimento do al eitamento exclusivo que, mesmo com suas vantagens e diversos recursos desenvolvidos parafacilitá-lo, éuma prática pouco valorizada no Brasil. Em 2001, os indicadores do Sistema de Informação deAtenção Básica (SI AB) demonstraram que 0 índice de al eitamento materno exclusivo foi de $63,3 \%$ no Brasil e $49,9 \%$ no Estado de Alagoas ${ }^{17}$. 0 fonoaudiólogo, inserido numa equipe interdisciplinar, também é responsável pela mudança desse quadro.

$\mathrm{Na}$ enfermaria, a fonoaudiologia, junto com outras profissões (pediatria, enfermagem, psicologia, terapia ocupacional, serviço social efisioterapia), realiza ações e estratégias voltadas ao estímulo ao aleitamento materno exclusivo, inclusive durante os horários da alimentação dos recém-nascidos, quando são identificadas difi- culdades esuporte específico érealizado pela equipe interdisci plinar. Essas ações, entre outras, asseguram a execução do modelo dialógico e participativo, no qual profissionais e usuários atuam como iguais, ainda que com papéis diferenciados. Inclusive, é possível perceber que algumas genitoras, já com os seus saberes transformados, começam a ser facilitadoras da promoção do aleitamento materno ${ }^{11}$.

As palestras educativas referentes à dinâmica da amamentação são ministradas pelas fonoaudiólogas, alunos do estágio curricular e enfermeiras conjuntamente. Consiste em mais um momento da comunicação dialogada desenvolvida nesse processo. Durante essa ação, abordase 0 aleitamento materno, introduzindo-sesimultaneamente 0 diálogo sobre técnicas facilitadoras da realização da pega efetiva do recém-nascido ao peito, levando-se em consideração o fato dequemães debebês prematuros ede baixo peso podem apresentar barreiras emocionais e psicológicas para iniciar e manter a lactação. Outros temas correlacionados também são abordados, como os hábitos orais deletérios. A família é envolvida em todas as atividades ${ }^{18}$.

Por meio do al eitamento materno, o bebêterá melhores condições de estimulação de seu sistema sensório-motor-oral, pois a extração do leite exige força muscular, aumentando assim a tonicidade muscular, que é questão importante para estimular as funções da fala, expiração e deglutição e para desenvolver as estruturas faciais e orais ${ }^{19}$.

Quando o aleitamento materno é substituído por mamadeiras e chupetas, o bebê, além de não ser devidamente estimulado na área sensório-motora, pode se desinteressar pela sucção do leitematerno. A partir destemomento, a musculatura perioral e de língua podem tornar-se hipotônicas, levando a uma alteração na deglutição normal e deformação da arcada dentária e palato, ocasionando mordida aberta frontal ou lateral ${ }^{20}$. As crianças que são amamentadas no seio possuem menor probabilidade de adquirir hábitos orais nocivos ${ }^{21}$.

Durante todo esse processo de promoção ao aleitamento exclusivo em prematuros, baseado no modelo dialógico, os aspectos culturais também são considerados, além deser necessário destacar que a prática do aleitamento materno também pode ser influenciada por esses aspectos.

No século $X X$, houve ascensão do aleitamento artificial e declínio do aleitamento materno, principalmenteem decorrência do avanço industrial e do aperfeiçoamento das técnicas de esteri- 
lização do leite de vaca, as quais propiciaram produção, em larga escala, de leite em pó industrializado. Somado a isso, a entrada da mulher no mercado de trabalho limitou a possibilidade de amamentação por seis meses, de forma exclusiva, o que favorece o desmame precoce ${ }^{22,23}$.

A partir de 1980, observa-se que a frequência decrianças em AM E (aleitamento materno exclusivo) no final do primeiro mês de vida foi mostrando expressiva elevação em relação ao mesmo período na década de setenta. Essa elevação no percentual de AM E ésemelhanteou maior queas frequências encontradas em outros estudos realizados no Brasil, nos Estados Unidos, na Noruega e na N ova Zelândia 24,25 .

Considerando, ainda, que o desmame pre coce é um importante problema de saúde pública em todo o mundo, relacionado a muitos fatores como idade materna, primiparidade, baixo nível de escolaridade, uso precoce de fórmulas lácteas e chupetas, trabal ho materno, urbanização, tabagismo, falta de incentivo da família e da sociedade, além de deficiências na atenção à saú$\mathrm{de}^{24} \mathrm{e}$ a condições de parto ${ }^{26}$, as características socioculturais, demográficas e epidemiológicas das genitoras atendidas no serviço é considerada, principalmente no que se refere à linguagem utilizada nas conversas e trocas de experiências entre a equipe de saúde e as puérperas.

Desta forma, inserida nas ações de educação em saúde, a atuação fonoaudiológica na enfermaria canguru da Maternidade Santa M ônica pode ser representada por cinco atividades:

a) estimulativa: busca atrair a genitora para participar do processo educativo;

b) exercitativa: condição para aquisição eformação de hábitos, assim como para a assimilação, construção e reconstrução de experiências;

c) orientadora: enfoca os aspectos de liberdade, autoridade, autonomia e independência;

d) didática: responsabiliza pela transmissão e veiculação dos conhecimentos;

e) terapêutica: permite retificar os eventuais descaminhos do processo educativo.

No ano de 2004, das 140 mães que receberam alta hospitalar, $100(71,43 \%)$ estavam amamentando exclusivamente. Em 2005, das 143 que receberam alta, 128 (89,51\%) estavam em aleitamento exclusivo e, no ano de 2006, das 210 altas hospitalares, 194 (92,3\%) genitoras amamentavam exclusivamente seus bebês ao seio.

0 aleitamento materno em prematuros, após inserção das facilitações baseadas na educação permanente, passou a ser discutido por toda a equipe. Cada profissional detecta aspectos perti- nentes a sua área e, a partir da discussão com a equipe, repassa o conhecimento das demandas apresentadas aos outros profissionais. Encontram-se também descritos na literatura baixos índices de desmame precoce na referida enfermaria canguru, no ano de $2006^{27}$.

Após alta hospitalar, o recém-nascido continua sendo acompanhado periodicamentedurante o primeiro ano de vida, no ambulatório de egresso. Toda a equipe encontra-se à disposição para quaisquer necessidades apresentadas pelo recém-nascido e sua família.

Implantar medidas deestímulo ao AM , como o contato precoce após o parto, a orientação de atitudes e comportamento dos familiares, a ampliação dos conhecimentos sobre leite materno e amamentação, o afastamento de hábitos nocivos e a facilitação no acesso aos serviços de saúde, constituem parteindispensável do esforço que deve ser dispendido por todos que cuidam da saúde da criança e da lactante ${ }^{28}$.

\section{Consideraçõesfinais}

No desenvolvimento de um processo de trabaIho baseado no pressuposto de que uma comunicação efetiva entrea equipe de saúde e os usuários do serviço de saúde é fundamental, percebeu-se que um dos principais passos para assistir a mãe que amamenta éavaliar seu sentimento a respeito do aleitamento.

Os encontros educativos devem ser oferecidos não apenas às nutrizes, mas aos familiares, que devem estar envolvidos ativamente na promoção do al eitamento materno exclusivo em prematuros. É importante haver um estreitamento de relacionamento entre a nutriz, sua família ea equipe interdisciplinar, para que haja segurança, confiança e consequente sucesso nesse processo.

Também é de fundamental importância que esse apoio continue após a alta hospitalar, o que aindaéum grande desafio na atuação fonoaudiológica (e da equipe que atua para a promoção do AM E). A proposta da atuação relatada está fundamentada na Política Nacional de Humanização da Assistência H ospitalar (PNHAH), preconizada pelo M inistério da Saúde, a qual objetiva trazer mudanças no modelo de atenção hospitalar no Sistema Ú nico de Saúde (SUS), buscando estratégias que possibilitem melhorias em relação ao contato humano entre os profissionais, pacientes e seus familiares, contribuindo para que as pressu postos de integralidade e equidade sejam fortalecidos. 
Uma educação em saúde ampliada inclui políticas públicas, ambientes apropriados e reorientação dos serviços de saúde para além dos tratamentos clínicos e curativos, assim como propostas pedagógicas li bertadoras comprometidas com o desenvolvimento da solidariedade e da cidadania, orientando-se para ações cuja essência está na mel horia da qualidade de vida. N estesentido, tem-se discutido sobre a consideração dos determinantes psicossociais e culturais nas práticas de educação em saúde. Propõe-se que estas sejam sensíveis às necessidades subjetivas e culturais dos usuários. Para tanto, reconhece-se a necessidade de abandonar estratégias comunicacionais informativas ea adoção de uma comunicação dialógica.
Espera-se que o conteúdo deste relato de experiência seja um estímulo ao debate, à reflexão, a propostas e ações para além dos processos de prevenção a doenças e promoção da saúde, ampliando-se na direção da formação de pessoas comprometidas com a luta contra as desigualdades sociais e em busca de encontros humanos mais solidários.

Considerar a educação em saúde como disciplina de ação si gnifica dizer queo trabalho édirigido para atuar sobre o conhecimento das pessoas, para que elas desenvolvam juízo crítico e capacidade de intervenção sobre suas próprias vidas e sobreo ambiente com o qual interagem $e$, assim, criarem condições para se apropriarem de sua própria existência.

\section{Colaboradores}

M CC Pessoa-Santana, AM M elo e EHAA Silva realizaram a pesquisa bibliográfica eredação inicial do artigo; BNG Goulart e BM Chiari supervisionaram a redação do relato do estudo, redação e revisão final do artigo. 


\section{Referências}

1. Marin CR, Chun RYS, Silva RC, Fedosse E, Leone Ili BS. Promoção da saúde em fonoaudiologia: ações coletivas em equipamentos de saúde e de educação. Rev Soc Bras Fonoaudiol. 2003; 8(1):35-41.

2. Freire RM. Fonoaudiologia em saúde pública. Rev Saude Publica 1992; 26:179-184.

3. Penteado RZ, Servilha EAM. Fonoaudiologia em saúde pública/coletiva: compreendendo prevenção e o paradigma da promoção da saúde. Distúrb Comun. 2004; 16(1):107-116.

4. Gonçalves MS, Tochetto TM, Primo MT. Fonoaudiologia e saúde coletiva: prioridades detectadas pelos usuários de unidades básicas de saúde. Rev Fonoaudiol Bras. 2005; 3(2):1-3.

5. Goulart BNG, Chiari BM. Construção e aplicação de indicadores de saúde na perspectiva fonoaudiológica - contribuição para reflexão. Rev Soc Bras Fonoaudiol. 2006;11(3):194-204.

6. Goulart BNG, Chiari BM. Humanização das práticas do profissional de saúde: contribuições para reflexão. Cien Saude Colet 2007; 1:405.

7. Vasconcelos EM. Redefinindo as práticas de saúde a partir da educação popular nos serviços de saúde. In: Vasconcelos EM, organizador. A saúde nas palavras e nos gestos: reflexões da rede de educação popular e saúde. São Paulo: Hucitec; 2001. p.11-19.

8. Mendes EV. Um novo paradigma sanitário: a produção social da saúde. In: Mendes EV. U ma agenda para a saúde. São Paulo: Hucitec; 1996. p. 233-300.

9. Buss PM. Promoção da saúde na infância e adolescência. Rev. Bras. Saúde M ater. Infant. 2001; 1:279282.

10. Costa M, López E. Educación para la salud. Madrid: Pirâmide; 1996. p. 25-58.

11. Briceño-Léon R. Siete tesis sobre la educación sanitaria para la participación comunitaria. Cad Saude Publica 1996; 12(1):7-30.

12. Smeke ELM, Oliveira NLS. Educação em saúde e concepções de sujeito. Inorganizador. A saúde nas palavras e nos gestos: reflexões da rede de educação popular e saúde. São Paulo: Hucitec; 2001. p. 115-136.

13. Chor D. Saúde pública e mudanças de comportamento: uma questão contemporânea. Cad Saude Publica 1999; 15(2): 423-425.

14. Ayres JRCM. Sujeito, intersubjetividade e práticas de saúde. Cien Saude Colet 2001; 6(1):63-72.

15. L'abbate S. Educação em saúde: uma nova abordagem. Cad Saude Publica 1994; 10(4):481-490.

16. Granzoto J, Bertoni A, Vecchi A, Rodrigues E. A importância do incentivo pré-natal na amamentação de primíparas. J. Pediatr. 1992; 68:34-37.

17. Brasil. Ministério da Saúde. Secretaria de Atenção Básica. Departamento de Atenção Básica. SIAB: indicadores 2001. 2ª ed. Brasília: M inistério da Saúde; 2003
18. Buhler KEB, Limongi SCO. O uso do copinho como método de alimentação de recém-nascidos pré-termo: revisão de literatura. Rev Soc Bras Fonoaudiol. 2004; 9(2):115-121.

19. Luiz Antonio Del Ciampo, Marcelo José Guimarães Junqueira, Rubens Garcia Ricco, Júlio Cesar Daneluzzi, I van Savioli Ferraz, Carlos Eduardo M artinelli Júnior. Tendência secular do al eitamento materno em uma unidade de atenção primária à saúde materno-infantil em Ribeirão Preto, São PauIo. Rev. Bras. Saúde M ater. Infant. 2006; 6(4):13-18.

20. Heath $A L$, Tuttle $C R$, Simons $M S$, Cleghorn $C L$, Parnell WR. A longitudinal study of breastfeeding and weaning practices during the first year of life in Dunedin, New Zealand. J Am Diet Assoc 2002; 102: 937-943.

21. Tu P. The effects of breastfeeding and birth spacing on child survival in China. Stud Fam Plann 1989; 20:332-342.

22. Issler H. Aleitamento materno: dificuldades e propostas. Rev Bras de M edicina 1987; 44:282-283.

23. Forman MR. Review of research of the factors associated with choice and duration of infant feeding in less-developed countries. Pediatrics 1984; 74:667-694. Li R, Zhao Z, Mokdad A, Barker L, Grummer-

24. Strawn L. Prevalence of breastfeeding in the United States: The 2001 National Immunization Survey. Pediatrics 2003; 111:1198-1202.

25. Escobar AMU, Ogawa AR, H iratsuka M, Kawashita MY, Teruya PY, Grisi S, Tomikawa SO. Aleitamento materno e condições sócioeconômico-culturais: fatores que levam ao desmame precoce. Rev. Bras. Saúde M ater. Infant. 2002; 2(3):253-261.

26. Alves AML, Silva, EHAA, Oliveira, AC. Desmame precoce em prematuros participantes do método mãe canguru. Rev Soc Bras Fonoaudiol. 2007; $12(1): 23-28$

27. Rezende MA. SOS respirador bucal: uma visão funcional e clínica da amamentação. Rev Latino-am Enfermagem 2004; 12(1):139.

28. Legovic M, Ostric L. The effects of feeding methods on the growth of the jaws in infants. J Dent Child 1991; 58(3):253-255.

Artigo apresentado em 13/12/2007

Aprovado em 29/10/2008

Versão final apresentada em 14/11/2008 